University of Texas at El Paso

ScholarWorks@UTEP

\title{
Is "No Trade Theorem" Really a Paradox: Analysis Based on Decision Theory
}

Laxman Bokati

The University of Texas at El Paso, Ibokati@miners.utep.edu

Vladik Kreinovich

The University of Texas at El Paso, vladik@utep.edu

Follow this and additional works at: https://scholarworks.utep.edu/cs_techrep

Part of the Applied Mathematics Commons, and the Computer Sciences Commons

Comments:

Technical Report: UTEP-CS-19-29

Published in Applied Mathematical Sciences, 2019, Vol. 13, No. 8, pp. 397-404.

\section{Recommended Citation}

Bokati, Laxman and Kreinovich, Vladik, "Is "No Trade Theorem" Really a Paradox: Analysis Based on Decision Theory" (2019). Departmental Technical Reports (CS). 1309.

https://scholarworks.utep.edu/cs_techrep/1309

This Article is brought to you for free and open access by the Computer Science at ScholarWorks@UTEP. It has been accepted for inclusion in Departmental Technical Reports (CS) by an authorized administrator of ScholarWorks@UTEP. For more information, please contact Iweber@utep.edu. 


\title{
Is "No Trade Theorem" Really a Paradox: Analysis Based on Decision Theory
}

\author{
Laxman Bokati and Vladik Kreinovich \\ Computational Science Program \\ University of Texas at El Paso \\ El Paso, Texas 79968, USA \\ lbokati@miners.utep.edu,vladik@utep.edu
}

\begin{abstract}
One of the challenges in foundations of finance is the so-called "no trade theorem" paradox: if an expert trader wants to sell some stock, that means that this trader believes that this stock will go down; however, the very fact that another expert trader is willing to buy it means that this other expert believes that the stock will go up. The fact that equally good experts have different beliefs should dissuade the first expert from selling - and thus, trades should be very rare. However, in reality, trades are ubiquitous. In this paper, we show that a detailed application of decision theory solves this paradox and explains how a trade can be beneficial to both seller and buyer. This application also explains a known psychological fact - that depressed people are usually more risk-averse.
\end{abstract}

\section{Formulation of the Problem}

"No trade theorem" paradox. When a bank or a hedge fund wants to buy a stock, this means that professionals running this financial institution believe that, in the future, this stock will increase in price. This makes perfect sense until we realize that for this institution to be able to buy this stock, some other institution needs to be willing to sell it at this price - which means that professionals running that other instutition must believe that, in the future, this stick will decrease in price.

Stock market is not a game for amateurs, serious agents buying and selling stock are smart experts who know what they are doing and who, in the past, have shown a good intuition about future stock values. So, even when such an expert initially thinks that this stock will increase in price, the very fact that this stock is available for sale means that another expert has an exactly opposite belief. This should, in many cases, dissuade the first expert from his or her original belief.

Similarly, an expert who is initially eager to sell, i.e., who initially believes 
that this stock will decrease in price, should be dissuaded by the presence of similarly qualified experts who are willing to buy, i.e., who believe that this stock will increase in price.

If we follow this logic, then very few agents will be trading stocks - but in reality, the trading volume is very high, every second, a huge amount of stocks change hands. This paradoxical behavior is known as a "no trade theorem"; see, e.g., $[8,12]$.

What we do in this paper. In this paper, we use decision making to show that in reality, trading makes perfect sense if we take into account different risks associated with different stocks.

Our explanation also explains another empirical phenomenon, a phenomenon from psychology - that depressed people are more risk-averse.

\section{Decision Theory: A Brief Reminder}

What is traditional decision theory. Traditional decision theory (see, e.g., $[1,4,7,10,11])$ described preferences of rational agents, i.e., e.g., agents that when preferring $A$ to $B$ and $B$ to $C$ would always prefer $A$ to $C$.

Comment. It is well known that real agents are not perfectly rational (see, e.g., $[2,6]$ ), for the simple reason that our ability to process information and select an optimal decision is bounded. However, in many cases, traditional decision theory still provides a very good picture of human behavior.

The notion of utility. To describe the preferences of such an agent, we can select two alternatives:

- a very bad one $A_{-}$that is much worse than this agent will actually encounter, and

- a very good one $A_{+}$that is much better than this agent will actually encounter.

For each value $p$ from the interval $[0,1]$, we can form a lottery $L(p)$ in which we get $A_{+}$with probability $p$ and $A_{-}$with the remaining probability $1-p$.

When $p$ is close to 1 , this means that we are almost certainly getting a very good deal. So, for any realistic option $A$, the corresponding lottery $L(p)$ is better than $A$ : $A<L(p)$. Similarly, when $p$ is close to 0 , this means that we are almost certainly getting a very bad deal, so $L(p)<A$. There should be a threshold $u$ at which the preference $L(p)<A$ corresponding to smaller probabilities $p$ is replaced by an opposite preference $A<L(p)$. In other words, we should have:

- $L(p)<A$ for all $p<u$ and

- $A<L(p)$ for all $p>u$.

This threshold value is called the utility of the alternative $A$; it is denoted by $u(A)$. 
The above two conditions means that, in a certain reasonable sense, the original alternative $A$ is equivalent to the lottery $L(u(A))$ corresponding to the probability $u(A): A \equiv L(u(A))$.

A rational agent should maximize utility. Of course, the larger the probability of getting a very good outcome $A_{+}$, the better. Thus, among several lotteries $L(p)$, we should select the one for which the probability $p$ of getting $A_{+}$is the largest. Since each alternative $A$ is equivalent to the corresponding lottery $L(u(A))$, this implies that we should select the alternative with the largest possible value of utility.

Main conclusion of traditional decision theory: a rational agent must maximize expected utility. In practice, we rarely know the consequences of each action. At best, we know possible outcomes $A_{1}, \ldots, A_{n}$, and their probabilities $p_{1}, \ldots, p_{n}$. Since each alternative $A_{i}$ is equivalent to a lottery $L\left(u\left(A_{i}\right)\right)$ in which we get $A_{+}$with probability $u\left(A_{i}\right)$ and $A_{-}$with the remaining probability $1-u\left(A_{i}\right)$, the whole action is equivalent to a two-stage lottery in which:

- first, we select one of the $n$ alternatives $A_{i}$ with probability $p_{i}$, and

- then, depending on which alternative $A_{i}$ we selected on the first stage, we select $A_{+}$with probability $u\left(A_{i}\right)$ and $A_{-}$with the remaining probability $1-u\left(A_{i}\right)$.

As a result of this two-stage lottery, we get either $A_{+}$or $A_{-}$. The probability $u$ of getting $A_{+}$can be computed by using the formula of complete probability, it is equal to

$$
u=p_{1} \cdot u\left(A_{1}\right)+\ldots+p_{n} \cdot u\left(A_{n}\right) .
$$

This is exactly the formula for the expected value of the utility $u\left(A_{i}\right)$. Thus, the utility of each action to a person is equal to the expected value of utility.

Thus, according to the traditional decision theory, rational agents should select the alternative with the largest possible value of expected utility.

How utility is related to money. The dependence of utility of money is non-linear: namely, utility $u$ is proportional to the square root of the amount $m$ of money $u=c \cdot \sqrt{m}$; see [2] and references therein.

Comment. This empirical fact can be explained. For example, the non-linear character of this dependence is explained, on a commonsense level, in [3], while the square root formula can also be explained - but it requires more mathematical analysis; see, e.g., [6]. In the current paper, we simply take this fact as a given, and use it to explain the "no trade theorem" paradox.

How to compare current and future gains: discounting. How can we compare current and future gains? If we have an amount $m$ of money now, then we can place it in a bank and get the same amount plus interest, i.e., get the new amount $m^{\prime} \stackrel{\text { def }}{=}(1+i) \cdot m$ in a year, where $i$ is the interest rate. Thus, the amount $m^{\prime}$ in a year is equivalent to the value $m=q \cdot m^{\prime}$ now, where $q \stackrel{\text { def }}{=} 1 /(1+i)$. 
This reduction of future gains - to make them comparable to current gains - is known as discounting.

\section{Analysis of the Problem and the Resulting Explanation of the "No Trade Theorem" Para- dox}

Towards formulation of the problem in precise terms. Let us assume that the person originally had the amount $M$ of money. This person is thinking of possibly buying a stock which costs $s$.

Let us also assume an ideal situation, in which everyone has the same information about the future value of this stock, namely, everyone knows the probability distribution of its next year's gain. In particular, everyone knows the mean $m^{\prime}$ and the standard deviation $\sigma^{\prime}$ of this future gain. After discounting, we get the mean $m=q \cdot m^{\prime}$ and the standard deviation $\sigma=q \cdot \sigma^{\prime}$ of the equivalent current gain.

Let us also make a realistic assumption that the price $s$, the mean $m$, and the standard deviation $\sigma$ are much smaller than the current money amount $M$. In other words, we assume that we are talking about a usual trade, not about extreme situations in which a person gambles his or her whole fortune by investing it all in a seemingly attractive stock.

Analysis of the problem. Let us denote the difference between the actual (discounted) value $v$ of the stock and its mean value $m$ by $\Delta v \stackrel{\text { def }}{=} v-m$. By definition of the mean, we have $m=E[v]$, where $E[\cdot]$ denoted the mean value. Thus, the mean value of $\Delta v$ is $0: E[\Delta v]=0$.

The mean value of $(\Delta v)^{2}$ is, by definition, equal to $\sigma^{2}: E\left[(\Delta v)^{2}\right]=\sigma^{2}$.

In these terms, the (discounted) future gain is equal to $v=m+\Delta v$. The discounted future amount of money can be obtained if we take the original amount $M$, subtract the cost $s$ of the stock, and add the gained value $v=$ $m+\Delta v ;$ as a result, we get the value $M-s+m+\Delta v$.

The utility is proportional to the square root of money. We can always select a unit of utility so that utility will be exactly equal to the square root of money. In this case, the original utility is $u=\sqrt{M}$, and the discounted future utility corresponding to buying a stock is equal to $u=\sqrt{M-s+m+\Delta v}$.

As we have mentioned in the previous section, a rational person should select the alternative with the largest possible value of expected utility $E[u]$. Thus, for the agent, it makes sense to buy the stock if

$$
E[u]=E[\sqrt{M-s+m+\Delta v}]>\sqrt{M} .
$$

If we have a reverse inequality, then, as one can easily see, it is beneficial for this person to sell this stock. So, to decide whether it is beneficial for a person to sell or buy the stock, we need to estimate the value $E[u]=E[\sqrt{M-s+m+\Delta v}]$ of the expected utility. 
Estimating the value of the expected utility. We assumed that the values $m, s$, and $\sigma$ are much smaller than $M$. Thus, the corresponding random value $\Delta v$ is also much smaller than $M$. So, we can expand the expression $\sqrt{M-s+m+\Delta v}$ in Taylor series in terms of $s, m$, and $\Delta v$, and keep only linear and quadratic terms in this expansion. As a result, we get the following expression:

$\sqrt{M-s+m+\Delta v}=\sqrt{M}+\frac{1}{2 \sqrt{M}} \cdot(-s+m+\Delta v)-\frac{1}{4 \cdot M^{3 / 2}} \cdot(-s+m+\Delta v)^{2}$.

If we open the parentheses and take into account that $E[\Delta v]=0$ and $E\left[(\Delta v)^{2}\right]=$ $\sigma^{2}$, we conclude that the expected utility of buying the stock is equal to

$$
E[u]=\sqrt{M}+\frac{1}{2 \sqrt{M}} \cdot(m-s)-\frac{1}{4 \cdot M^{3 / 2}} \cdot\left((m-s)^{2}+\sigma^{2}\right) .
$$

Thus, this value is larger than the original utility $\sqrt{M}$ if and only if $E[u]-\sqrt{M}>$ 0 , i.e., if and only if

$$
\frac{1}{2 \sqrt{M}} \cdot(m-s)-\frac{1}{4 \cdot M^{3 / 2}} \cdot\left((m-s)^{2}+\sigma^{2}\right)>0 .
$$

Multiplying both sides by $4 \cdot M^{3 / 2}$, we get an equivalent inequality

$$
2 M \cdot(m-s)-\left((m-s)^{2}+\sigma^{2}\right)>0,
$$

i.e., equivalently, $2 M \cdot(m-s)>\left((m-s)^{2}+\sigma^{2}\right)$ and

$$
M>M_{0} \stackrel{\text { def }}{=} \frac{(m-s)^{2}+\sigma^{2}}{2(m-s)} .
$$

This explains the "no trade theorem" paradox. For the same stock with the same information about its future gains, whether it is beneficial to buy it or sell it depends on the initial amount of money that a trader has:

- if the trader has a large amount of money $M$, then buying a stock whose expected benefits $m$ exceed the buying cost $s$ makes perfect sense, even when the risk $\sigma$ is reasonably high;

- on the other hand, if the trader has a not so large amount of money and the stock is risky, then for this trader, it makes sense to sell this stock.

For this stock, for almost all traders (with a rare exception of a trader whose current amount is exactly $M_{0}$ ), it is either beneficial to buy (if $M>M_{0}$ ) or to sell (if $M<M_{0}$ ).

Thus, for the same stock, with the same information, we always have many traders for whom it is beneficial to buy, and we have many traders for whom it is beneficial to sell. This explains the ubiquity of trading. 


\section{Auxiliary Result: Decision Theory Explains Why Depressed People Are More Risk-Averse}

Empirical fact. It has been observed that depressed people are more riskaverse, i.e., they are less willing to make decisions involving risks; see, e.g., [5, 9].

Our explanation. Each risky decision is described by the same formulas as a particular case of buying-a-stock risky decision: we may gain something, we may lose something, all we know is the probability distribution of the corresponding gains and losses.

Thus, to decide when it is beneficial to participate in a risky activity, we can use the same formulas as above - the only difference is that instead of just money amount $M$ and the corresponding initial utility $u_{0}=\sqrt{M}$, we can take into account different things that affect the person's utility. In terms of utility $u_{0}$, the inequality (1) - that describes when it is beneficial for a person to engage in a risky behavior - takes the form

$$
u_{0}^{2}>M_{0} \stackrel{\text { def }}{=} \frac{(m-s)^{2}+\sigma^{2}}{2(m-s)} .
$$

This formula says that when the initial value of the utility $u_{0}$ is small, risky behavior - with large $\sigma$ - is not beneficial. And this is exactly what depression means in decision-theoretic terms: that a person is not very happy, i.e., that the corresponding utility value $u_{0}$ is small.

Thus, our decision-theoretic analysis explains the above-mentioned psychological phenomenon.

\section{Acknowledgments}

This work was supported in part by the US National Science Foundation grant HRD-1242122 (Cyber-ShARE Center of Excellence).

\section{References}

[1] P. C. Fishburn, Utility Theory for Decision Making, John Wiley \& Sons Inc., New York, 1969.

[2] D. Kahneman, Thinking, Fast and Slow, Farrar, Straus, and Giroux, New York, 2011.

[3] O. Kosheleva, M. Afravi, and V. Kreinovich, "Why utility non-linearly depends on money: a commonsense explanation", Proceedings of the 4 th International Conference on Mathematical and Computer Modeling, Omsk, Russia, November 11, 2016, pp. 13-18. 
[4] V. Kreinovich, "Decision making under interval uncertainty (and beyond)", In: P. Guo and W. Pedrycz (eds.), Human-Centric Decision-Making Models for Social Sciences, Springer Verlag, 2014, pp. 163-193.

[5] Y. Leykin, C. S. Roberts, and R. J. DeRubeis, "Decision-making and depressive symptomatology", Cognitive Therapy and Research, 2011, Vol. 35, pp. 333-341.

[6] J. Lorkowski and V. Kreinovich, Bounded Rationality in Decision Making Under Uncertainty: Towards Optimal Granularity, Springer Verlag, Cham, Switzerland, 2018.

[7] R. D. Luce and R. Raiffa, Games and Decisions: Introduction and Critical Survey, Dover, New York, 1989.

[8] P. Milgrom and N. Stokey, "Information, trade and common knowledge", Journal of Economic Theory, 1982, Vol. 26, No. 1, pp. 17-27.

[9] F. C. Murphy, J. S. Rubinsztein, A. Michael, R. D. Rogers, T. W. Robbins, E. S. Paykel, and B. J. Sahakian, "Decision-making cognition in mania and depression", Psychological Medicine, 2001, Vol. 31, pp. 679-693.

[10] H. T. Nguyen, O. Kosheleva, and V. Kreinovich, "Decision making beyond Arrow's 'impossibility theorem', with the analysis of effects of collusion and mutual attraction", International Journal of Intelligent Systems, 2009, Vol. 24, No. 1, pp. 27-47.

[11] H. Raiffa, Decision Analysis, McGraw-Hill, Columbus, Ohio, 1997.

[12] R. H. Thaler, Misbehaving: The Making of Behavioral Economy, W. W. Norton \& Co., New York, 2015. 